\title{
An analysis of microbiota-targeted therapies in patients with avian influenza virus subtype H7N9 infection
}

Haifeng Lu ${ }^{1,2+}$, Chunxia Zhang ${ }^{3 \dagger}$, Guirong Qian ${ }^{1,2}$, Xinjun Hu ${ }^{1,2}$, Hua Zhang ${ }^{1,2}$, Chunlei Chen ${ }^{1,2}$, Weifeng Liang ${ }^{1,2}$, Hainv Gao ${ }^{1,2}$, Yunmei Yang ${ }^{3^{*}}$ and Lanjuan $\mathrm{Li}^{1,2^{*}}$

\begin{abstract}
Background: Selective prophylactic decontamination of the digestive tract is a strategy for the prevention of secondary nosocomial infection in patients with avian influenza virus subtype H7N9 infection. Our aim was to summarize the effectiveness of these therapies in re-establishing a stable and diverse microbial community, and reducing secondary infections.
\end{abstract}

Methods: Comprehensive therapies were dependent on the individual clinical situation of subjects, and were divided into antiviral treatment, microbiota-targeted therapies, including pro- or pre-biotics and antibiotic usage, and immunotherapy. Quantitative polymerase chain reaction and denaturing gradient gel electrophoresis (DGGE) were used for real-time monitoring of the predominant intestinal microbiome during treatment. Clinical information about secondary infection was confirmed by analyzing pathogens isolated from clinical specimens.

Results: Different antibiotics had similar effects on the gut microbiome, with a marked decrease and slow recovery of the Bifidobacterium population. Interestingly, most fecal microbial DGGE profiles showed the relative stability of communities under the continual suppression of the same antibiotics, and significant changes when new antibiotics were introduced. Moreover, we found no marked increase in C-reactive protein, and no cases of bacteremia or pneumonia, caused by probiotic use in the patients, which confirmed that the probiotics used in this study were safe for use in patients with H7N9 infection. Approximately $72 \%$ of those who subsequently suffered exogenous respiratory infection by Candida species or multidrug-resistant Acinetobacter baumannii and Klebsiella pneumoniae were older than 60 years. The combination of probiotics and prebiotics with antibiotics seemed to fail in these patients.

Conclusions: Elderly patients infected with the influenza A (H7N9) virus are considered a high-risk group for developing secondary bacterial infection. Microbiota restoration treatment reduced the incidence of enterogenous secondary infection, but not exogenous respiratory infection. The prophylactic effects of microbiota restoration strategies for secondary infection were unsatisfactory in elderly and critically ill patients.

Keywords: H7N9, Intestinal microbiota, Probiotics, Microbiota restoration treatment, Quantitative PCR

\footnotetext{
* Correspondence: ymyang@zju.edu.cn; ljil@zju.edu.cn

${ }^{\dagger}$ Equal contributors

${ }^{3}$ Department of Geriatrics, the First Affiliated Hospital, College of Medicine,

Zhejiang University, 79 Qingchun Road, Hangzhou 310003, P.R. China

${ }^{1}$ State Key Laboratory Diagnosis and Treatment for Infectious Disease,

Collaborative Innovation Center for Diagnosis and Treatment of Infectious

Diseases, the First Affiliated College of Medicine, Zhejiang University, 79

Qingchun Road, Hangzhou 310003, China

Full list of author information is available at the end of the article
} 


\section{Background}

Forty cases of confirmed avian influenza virus subtype H7N9 infection were treated at the First Affiliated Hospital of Zhejiang University (China) during March-April 2013. Most of the patients were critically ill and required admission to an intensive care unit [1], wheretreatment can be divided into antiviral treatment, microbiota-targeted therapies, and immunotherapy. Secondary invasive bacterial infections associated with H7N9 infection can cause severe and fatal complications, and appropriate empirical antibiotic treatment for hospital-acquired bacterial infections is required. The human gut harbors a complex microbiome that plays a fundamental role in host health through its involvement in nutritional, immunological, and physiological functions [2]. Reciprocal interactions between intestinal microbiota and the human mucosal immune response influence the development of disease through activation of innate and adaptive immune responses [3]. Antibiotics have a profound disruptive effect on the intestinal microbiome [4], which is frequently accompanied by colonization of pathogenic microbes and dysbiosis of the host immune system, contributing to the development of potentially serious diseases $[5,6]$. Therefore, microbiota restoration strategies, which allow beneficial bacteria to thrive, eliminate colonization by opportunistic pathogens, and enhance resistance to intestinal colonization, are required for treatment of H7N9 infection. Intestinal colonization resistance is defined as the resistance to colonization by ingested bacteria or inhibition of overgrowth of resident bacteria normally present at low levels within the intestinal tract [7]. Probiotics and prebiotics have a beneficial effect on regeneration and restoration of microbiome homeostasis [8]. Additionally, more recent data show that probiotics can modulate host immunoregulation, alleviate intestinal inflammation, normalize gut mucosal dysfunction, and downregulate hypersensitivity reactions through control of proinflammatory and anti-inflammatory cytokines [9]. Theoretically, targeted microbiota-regulating treatment could prevent or alleviate the complications of $\mathrm{H} 7 \mathrm{~N} 9$ infection.

CBM588 (Clostridium butyricum 588 strain MIYA-BM tablets; Miyarisan Pharmaceutical, Tokyo, Japan) prevents antibiotic-associated diarrhea [10] and has antiinflammatory effects in the colon as a result of butyric acid production [11]. We retrospectively assessed the effects of microbiota restoration strategies on restoring intestinal homeostasis under antibiotic exposure, and on reducing the risk of secondary infection, by real-time monitoring of the shift in predominant intestinal bacteria using quantitative polymerase chain reaction
(qPCR) and PCR-based denaturing gradient gel electrophoresis (PCR-DGGE).

\section{Methods}

\section{Patients and stool specimen sampling}

The study was conducted between 1 April and 10 May 2013 in the First Affiliated Hospital, College of Medicine of Zhejiang University, China. Approval for the collection of patient stool samples was obtained from the ethical board of the hospital. All patients voluntarily joined the study and gave their informed consent. We prospectively studied all consecutive, nonselected inpatients who had episodes of influenza virus H7N9 infection. Patients were diagnosed according to the criteria of the Centers for Disease Control, USA, in 2013 [12]. Stool samples were collected daily, and intestinal microecology was investigated in all specimens. Patients were excluded if they provided fewer than four stool specimens during the follow-up period. The follow-up for each patient ended once their major method of feeding changed, for example when patients that had been fed using a nasogastric tube graduated to autonomous feeding. Fecal samples were collected in sterile bags, refrigerated, and immediately taken to the laboratory where they were aliquoted into 200-mg samples, frozen in liquid nitrogen, and stored at $-75^{\circ} \mathrm{C}$. Twenty-five patients completed sampling, and a total of 205 specimens were subject to qPCR and DGGE analysis. Eleven of these patients had secondary infection, of which, eight patients were over 60 years of age. Patient characteristics were collected from medical records and are in shown Table 1. Patients were classified into five groups according to microbiota-targeted treatment: Group A, two patients without any microbiota-targeted treatment during follow-up; Group B, one patient with one antibiotic only; Group C, six patients with probiotic only; Group $D$, eight patients with one antibiotic and one probiotic; and Group E, eight patients with two or more antibiotics and probiotics or prebiotics. Groups A-C had mild disease and could feed independently, whereas Groups $\mathrm{D}$ and $\mathrm{E}$ were critically ill and required nasogastric feeding. Twenty-five healthy age- and sex-matched controls (Table 1) were enrolled and provided only one stool sample. The participants did not have any organic diseases, none withdrew or were omitted from the study, and none received antibiotics, probiotics, or prebiotics during the sampling period.

\section{DNA extraction}

DNA was extracted from the feces and bacterial precipitates using a Qiagen Stool Kit (Qiagen, Hilden, Germany) with a modified protocol for cell lysis [13]. DNA integrity was confirmed by agarose gel electrophoresis and ultraviolet photography with ethidium bromide staining. 
Table 1 Characteristics of all subjects andusage of microbiota-targeted agents in secondary infection and non-secondary infection groups of H7N9 patients

\begin{tabular}{|c|c|c|c|}
\hline Characteristics & $\begin{array}{l}\text { Secondary infection group } \\
\qquad(n=11)\end{array}$ & $\begin{array}{l}\text { Non-secondary infection group } \\
\qquad(n=14)\end{array}$ & $\begin{array}{l}\text { Healthy controls } \\
\quad(n=25)\end{array}$ \\
\hline Median age (years) & 64.5 & 48 & 58 \\
\hline Male:female $(n)$ & $9: 2$ & $8: 6$ & $17: 8$ \\
\hline Median hospital stay (days) & 21.5 & 13 & $-{ }^{a}$ \\
\hline \multicolumn{4}{|l|}{ Primary blood parameters at first follow-up: } \\
\hline Mean WBC (SD, $10^{9}$ cells per liter) & $8.0(3.5)$ & $4.6(3.3)$ & $5.7(1.3)$ \\
\hline Mean CRP (SD, mg per liter) & $61.7(72.3)$ & $42.8(53.2)$ & - \\
\hline ALT median $\left(25^{\text {th }}-75^{\text {th }}\right.$ percentile, units per liter) & $38(25-50)$ & $44(19.5-114)$ & $16(12.5-21)$ \\
\hline Antimicrobial usage: & & & - \\
\hline Cephalosporins $(n)$ & 7 & 9 & - \\
\hline Carbapenems $(n)$ & 6 & 1 & - \\
\hline$\beta$-Lactamase inhibitors and cephalosporins ( $n$ ) & 4 & 3 & - \\
\hline$\beta$-Lactamase inhibitors and penicillins ( $n$ ) & 9 & 5 & - \\
\hline Quinolones (n) & 10 & 8 & - \\
\hline Macrolides ( $n$ ) & 4 & 1 & - \\
\hline Vancomycin $(n)$ & 5 & 0 & - \\
\hline Tigecycline $(n)$ & 4 & 0 & - \\
\hline Oxazolidinones ( $n)$ & 3 & 0 & - \\
\hline Phosphonomycin (n) & 1 & 1 & - \\
\hline Antifungalagents $(n)$ & 1 & 0 & - \\
\hline Micro-ecological-targeted agents & & & - \\
\hline CBM588 (n) & 9 & 11 & - \\
\hline Bacillussubtilis- and E. faecium-coated enteric capsules ( $n$ ) & 1 & 1 & - \\
\hline Lactulose $(n)$ & 4 & 0 & - \\
\hline Infection with & & & - \\
\hline Canidia $(n)$ & 7 & 0 & - \\
\hline Klebsiella pneumoniae, $n$ & 6 & 0 & - \\
\hline Acinetobacter baumanii ( $n$ ) & 5 & 0 & - \\
\hline Pseudomonas (n) & 5 & 0 & - \\
\hline Flavobacterium indologenes ( $n$ ) & 1 & 0 & - \\
\hline Staphylococcus (n) & 2 & 0 & - \\
\hline Infections with mixed culture (n) & 7 & 0 & - \\
\hline Diarrhea (n) & 3 & 0 & - \\
\hline
\end{tabular}

Abbreviations: WBC white blood cell, SD standard deviation, CRP C-reactive Protein, ALT alanine aminotransferase, CBM588, Clostridium butyricum 588 strain MIYA-BM tablets.

${ }^{\mathrm{a}}$ Not applicable.

Qualitative and quantitative PCR to detect predominant intestinal bacterial population

Primers used were described in an earlier study [13] (Table 2). All oligonucleotide primers were synthesized by Takara (Dalian, China). Quantitative PCR was performed using a ViiA 7 Real-Time PCR System (Applied Biosystems, Foster City, CA, USA). Amplification reactions contained $10 \mu \mathrm{l}$ of SYBR Green PCR Master Mix (Applied Biosystems, Warrington, UK), $300 \mathrm{nM}$ each primer, and $300 \mathrm{ng}$ of the respective crude template DNA or $1 \mu \mathrm{L}$ of water (negative control) in a final volume of $20 \mu \mathrm{L}$. Each reaction was performed in duplicate, and a $\Delta C(\mathrm{t})<0.5$ between duplicates was required. Amplifications were performed with the following temperature profiles: one cycle at $95^{\circ} \mathrm{C}$ for $3 \mathrm{~min}$, followed by 40 cycles of $95^{\circ} \mathrm{C}$ for $30 \mathrm{~s}$, annealing for $40 \mathrm{~s}$, and $72^{\circ} \mathrm{C}$ for $30 \mathrm{~s}$. Fluorescence was measured at $80^{\circ} \mathrm{C}$ for $10 \mathrm{~s}$ after the extension phase of each cycle to avoid interference from primer dimers, secondary structure, or 
Table 2 Primers used in this study

\begin{tabular}{|c|c|c|}
\hline Target group & Sequence $\left(5^{\prime}-3^{\prime}\right)$ & Annealing temperature $\left({ }^{\circ} \mathrm{C}\right)$ \\
\hline \multirow[t]{2}{*}{ Faecalibacterium prausnitzii } & GATGGCCTCGCGTCCGATTAG & 58 \\
\hline & CCGAAGACCTTCTTCCTCC & \\
\hline \multirow[t]{2}{*}{ Bifidobacterium genus } & GGGTGGTAATGCCGGATG & 59 \\
\hline & TAAGCCATGGACTITCACACC & \\
\hline \multirow[t]{2}{*}{ Lactic acid bacteria } & AGCAGTAGGGAATCTTCCA & 58 \\
\hline & ATTYCACCGCTACACATG & \\
\hline \multirow[t]{2}{*}{ Enterococcus faecalis } & AACCTACCCATCAGAGGG & 57 \\
\hline & GACGTTCAGTTACTAACG & \\
\hline \multirow[t]{2}{*}{ Enterobacteriaceae } & CATTGACGTTACCCGCAGAAGAAGC & 63 \\
\hline & CTCTACGAGACTCAAGCTTGC & \\
\hline \multirow[t]{2}{*}{ Bacteroides-Prevotella group } & GAAGGTCCCCCACATTG & 56 \\
\hline & CAATCGGAGTTCTTCGTG & \\
\hline
\end{tabular}

spurious priming. A final extension step of $72^{\circ} \mathrm{C}$ for $5 \mathrm{~min}$ was performed. The annealing and plate-reading temperatures for each primer pair are shown in Table 2. Following amplification, melting temperature analysis of PCR products was performed to determine the specificity of the PCR. The melting curves were obtained by slow heating at $0.1^{\circ} \mathrm{C} / \mathrm{s}$ from $65^{\circ} \mathrm{C}$ to $95^{\circ} \mathrm{C}$, with continuous fluorescence measurement.

The copy number of rDNA operons of targeted bacteria in crude DNA templates was determined by comparison with serially-diluted plasmid DNA standards run on the same plate. The plasmid DNA standards were made from known concentrations of plasmid DNA that contained the respective amplicon for each set of primers, according to Bartosch et al. [14]. Determination of the detection limit of the assays was performed as described previously [15].

\section{PCR amplification of the $16 \mathrm{~S}$ rDNA V3 region}

The V3 variable region of $16 \mathrm{~S}$ rDNA was amplified using a hot-start touchdown protocol with primers specific for conserved regions of the $16 \mathrm{~S}$ rRNA gene [16]. The reaction mixture contained $800 \mathrm{ng}$ of genomic DNA, 25 pmol of each primer, $0.2 \mu \mathrm{M}$ dNTPs, $1 \times$ Ex Taq buffer, and $2.5 \mathrm{U}$ of Ex Taq polymerase (Takara). The final volume of the reaction mixture was adjusted to $50 \mu \mathrm{L}$ with sterile deionized water. To minimize heteroduplex formation, five-cycle reconditioning PCR was conducted using $5 \mu \mathrm{L}$ of amplification mixtures in fresh reaction mixture, as previously described [17]. Thermal cycling was performed in a TProfessional Thermocycler (Biometra, Göttingen, Germany). Concentrations were measured using a NanoDrop ND1000 spectrophotometer (NanoDrop Technologies, Wilmington, DE, USA, USA).

\section{Denaturing gradient gel electrophoresis profiling}

Parallel DGGE analysis was performed using the DCode universal mutation detection system apparatus
(Bio-Rad, Hercules, CA, USA) with $16 \mathrm{~cm} \times 18 \mathrm{~cm} \times$ $1.5 \mathrm{~mm}$ gels. Sequence-specific separation of the PCR fragments was obtained in $8 \%$ (wt/vol) polyacrylamide (acrylamide-N, N'-bisacrylamide, 37.5:1 (wt/vol)) gels in $1 \times$ TAE buffer (40 mM Tris, $20 \mathrm{mM}$ glacial acetic acid, $1 \mathrm{mM}$ EDTA, pH 8.0). Denaturing gels contained a 35$75 \%$ gradient of urea and formamide, increasing in the direction of electrophoresis. A 100\% denaturing solution contained $40 \%$ (vol/vol) formamide and $7 \mathrm{M}$ urea. PCR fragments $(20 \mu \mathrm{L})$ were loaded onto gels and electrophoresis was conducted at a constant voltage of $70 \mathrm{~V}$ and a temperature of $60^{\circ} \mathrm{C}$ for approximately $16 \mathrm{~h}$. Following electrophoresis, gels were stained with SYBR green I (Sigma-Aldrich, Castle Hill, Australia) and photographed. Each DGGE gel included a standard reference (a randomly selected sample) in the middle and at both ends for digital gel normalization, and to allow comparison between gels.

DGGE profiles were digitally processed using BioNumerics software version 6.01 (Applied Maths, StMartens-Latem, Belgium) following the manufacturer's instructions. All profiles were compared using the bandmatching tool, and uncertain bands were included in the position tolerance settings. Bands were allocated according to the parameters of Joossens et al. [18]. Principal components analysis (PCA) analysis and DGGE Bands Sequencing were performed according to a previous study [19].

\section{Results}

Retrospective analysis of microbiota-targeted treatment and secondary infection

Early clinical experience with influenza virus H7N9 therapy showed that patients often developed acute exacerbation with bacterial superinfection. These serious complications could sometimes be fatal. Prophylactic selective decontamination of the digestive tract is suggested for increased 
survival of critically ill patients with H7N9 pneumonia. Therefore, all patients in our study received different broad-spectrum antibiotics in their district hospitals prior to transfer (Table 1 and Figure 1) to remove any pathogenic microorganisms. Most patients also received prophylactic selective decontamination of the digestive tract during the follow-up period. The antibiotics used in these patients were classified into nine groups according to

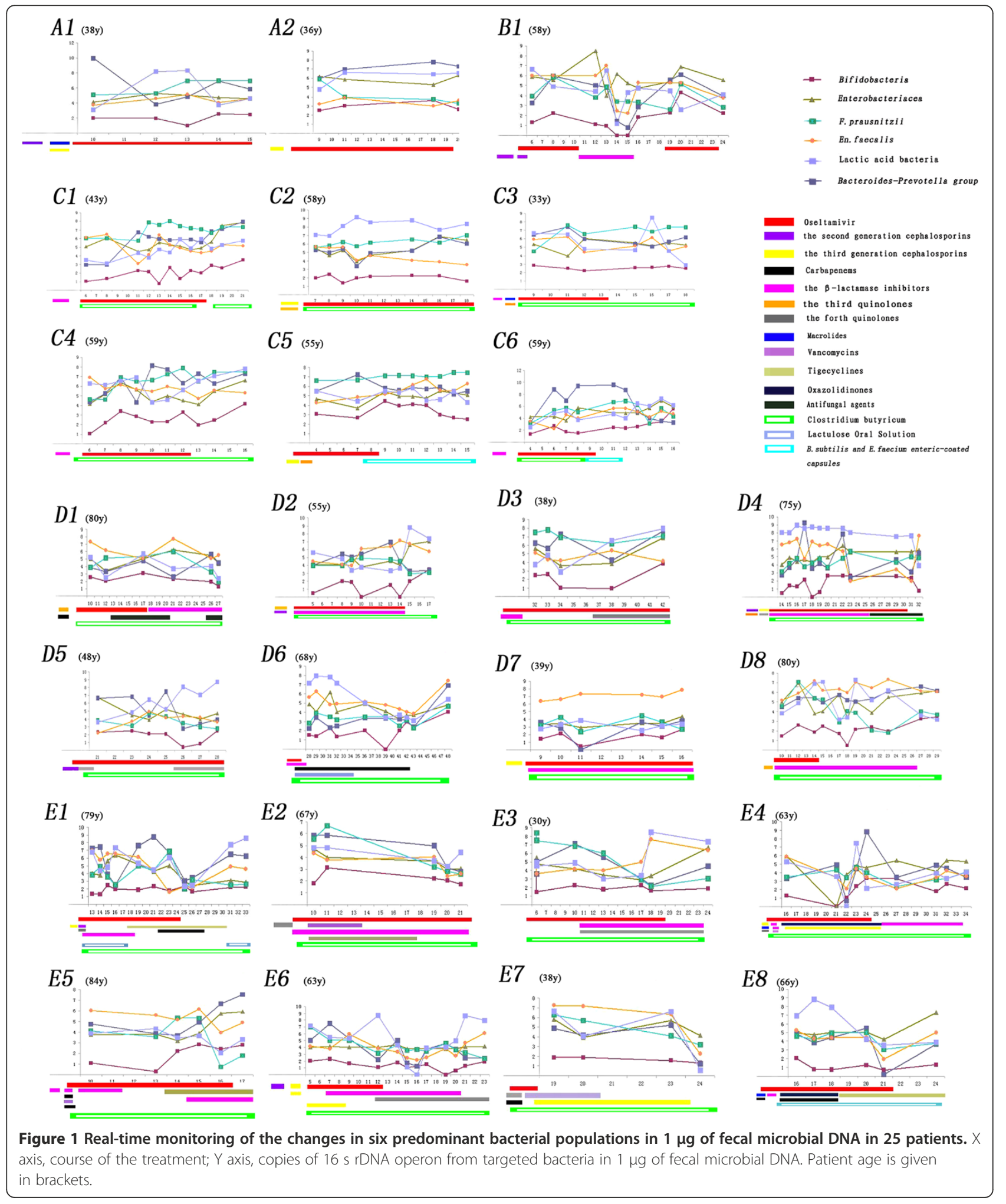


molecular structure: cephalosporins (cefuroxime, cefoxitin, cefmetazole, ceftazidime, cefotaxime, ceftriaxone, cefdinir, ceftizoxime, and cefepime), carbapenems (imipenem and meropenem), $\beta$-lactam and $\beta$-lactamase inhibitors (cefoperazone sodium and sulbactam sodium, piperacillintazobactam, piperacillin sodium, and sulbactam sodium), quinolones (moxifloxacin, ciprofloxacin, and levofloxacin), macrolides (azithromycin and roxithromycin), vancomycin, tigecycline, oxazolidinones (linezolid), phosphonomycin, and antifungal agents (fluconazole and caspofungin). Probiotics, including Clostrium butyricum-, Bacillus subtilis-, and Enterococcus faecium-coated enteric capsules, and prebiotics, such as lactulose, were used in most patients in consideration of impaired intestinal colonization resistance and disturbance of microbiota caused by the broad-spectrum antimicrobial agents. Probiotic compounds were administered as two tablets, three times per day (containing $\sim 10^{7} \mathrm{cfu} /$ tablet for CBM588 and $10^{8}$ cfu for B. subtilis and E. faecium capsules). All microbiota-targeted agents were taken as advised, and administered by an expert team according to patient health status, the targeted disease, and the recipient's perceived risks and willingness.
Eleven patients (C5, D1, D3, D6, E1, E2, and E4-E8) acquired secondary bacterial infections during their stay in the intensive care unit for treatment of critical pneumonia. Eight of the eleven patients (72\%) were older than 60 years of age. Pathogens were isolated from their clinical specimens. Six samples contained Klebsiella pneumoniae (isolated from sputum), seven had Candida species (isolated from sputum), five had Acinetobacter baumanii (isolated from sputum), five had Pseudomonas species (isolated from sputum), two had Staphylococcus species (isolated from blood), and one had Flavobacterium indologenes (isolated from blood). Seven had mixed bacterial infections. Three patients from group E suffered from antibiotic-associated diarrhea caused by Clostridium difficile, which was cultured from stool samples.

We also monitored the real time concentration of $\mathrm{C}$ reactive protein (CRP), an early indicator of infection or inflammation, in the blood of each patient. Results showed that CRP tended to decrease in most H7N9 patients, particularly those without secondary infections (Figure 2), which suggested that the probiotics used were safe and did not cause an inflammatory state.

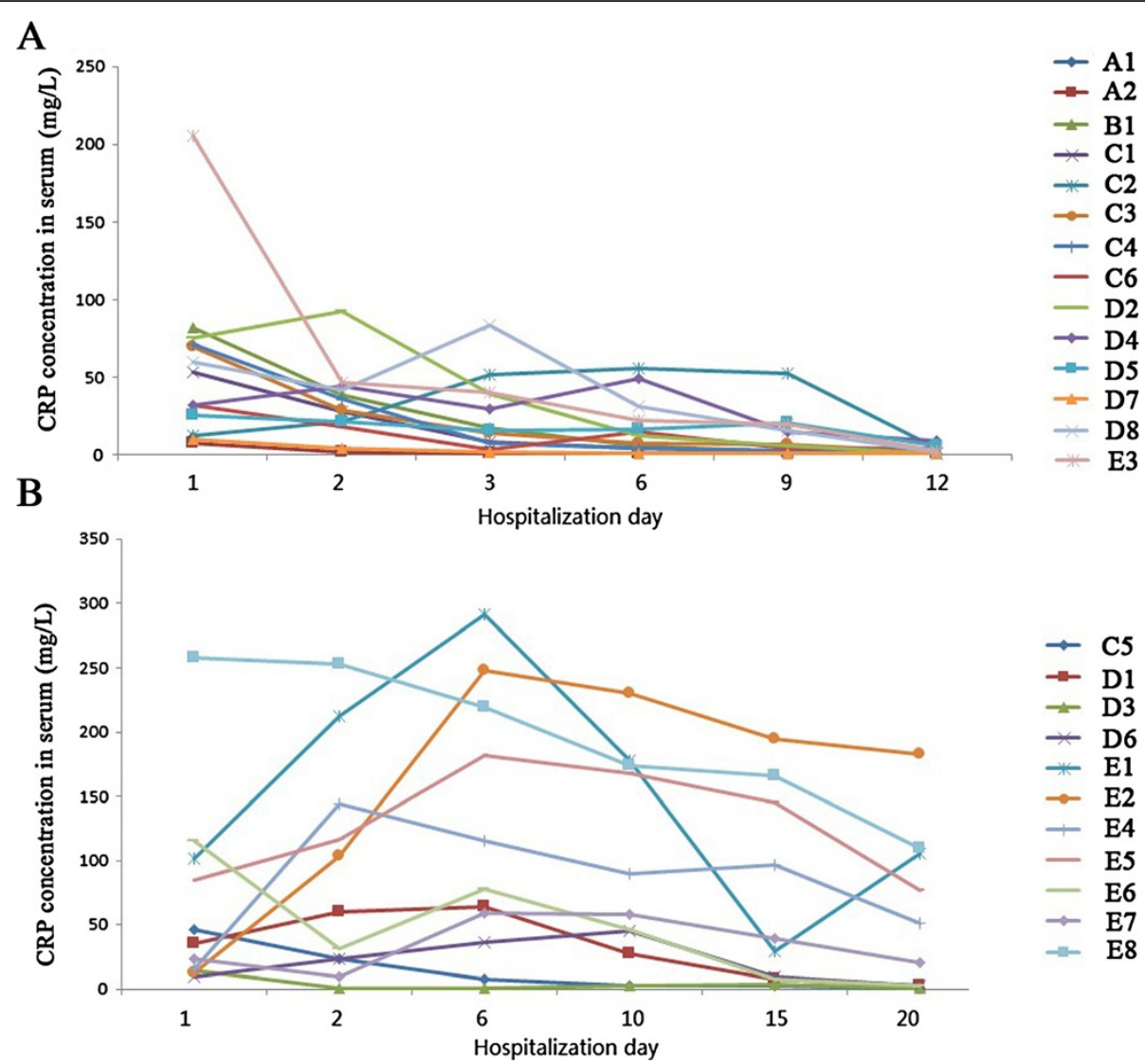

Figure 2 The concentrations of C-reactive protein (CRP) in blood (mg/L). A, Patients without secondary infection; B, patients with secondary infections. 


\section{Intestinal Bifidobacterium/Enterobacteriaceae ratio in patients with H7N9 infection}

All subjects were assessed for their intestinal Bifidobacterium/Enterobacteriaceae (B/E) ratio in the total fecal microbial DNA (Figure 3). B/E ratios of most healthy controls were $\geq 1$, except for four individuals aged $\geq 80$ years (red triangles in Figure 3) who had ratios $<1$. In contrast, the ratios of all patients were $<1$, and most were even smaller than in the elderly healthy controls.

\section{Real-time monitoring of the predominant intestinal microbiome}

The quantification data of six predominant intestinal bacteria, Bifidobacterium, Enterobacteriaceae, Bacteroides-Prevotella group, Faecalibacterium prausnitzii, Enterococcus faecalis, and lactic acid bacteria, were expressed as $\log _{10}$ $16 \mathrm{~S}$ rRNA gene copies in $1 \mu \mathrm{g}$ fecal DNA. The levels of these bacteria in healthy controls are shown in Figure 4.

Two patients, A1 and A2, only received antiviral treatment at the follow-up stage, and antimicrobial treatment in the early stage had significantly decreased the Bifidobacterium population (A1 and A2 in Figure 1). The influence of antibiotics on fecal bacteria was clearly demonstrated in Patient B1, who had a marked decrease in the six bacterial groups during a 5-day period of antibiotic treatment in the follow-up period (B1 in Figure 1). The levels of these populations largely rebounded after antibiotic treatment ended. Notably, this patient had an increase in Enterobacteriaceae when taking piperacillin sodium and sulbactam sodium, which suggested an increase in resistant strains that are present in small numbers in the healthy intestinal tract. Patients $\mathrm{C} 1-\mathrm{C} 6$ received microbiota restoration therapies $(\mathrm{C} 1-\mathrm{C} 6$ in
Figure 1) without any antibiotics after enrollment, and the population sizes of the six bacterial groups increased. However, the populations of Bifidobacterium, F. prausnitzii, and lactic acid bacteria showed slight variation in some cases. In particular, the six bacterial groups were relatively stable in Patient $\mathrm{C} 5$, who received $B$. subtilisand E. faecium-coated enteric capsules; however, Candida albicans was isolated from the sputum during follow-up. Bacillus subtilis and E. faecium capsules replaced CBM588 tablets for 2 days in Patient C6, and the six bacterial populations did not show substantial fluctuations at the termination of probiotic treatment. Data from eight patients in Group D who received one type of antibiotic and CBM588 tablets showed that CBM588 decreased the impact of the antibiotics, and increased the bacterial populationduring the period of continual suppression of the same antibiotic (D1-D8 in Figure 1). Additionally, the Bifidobacterium population generally trended upward in most patients in Group D. For critically ill patients in Group E (E1-E8 in Figure 1), the populations of the six bacterial groups fell to their lowest levels and then began to rebound. Most patients in Group E (7/8) had secondary infection and diarrhea during the follow-up period.

Figure 5 shows the predominant intestinal microbiome profiles of representatives of each group. The predominant intestinal microbiome of control subjects was more evenly distributed, with higher species richness, than that of patients. The predominant bands in the profiles of $\mathrm{B} 1$ and $\mathrm{A} 1$ were distributed in the region where the denaturant concentrations had a range of $37-57 \%$, but those dosed with $C$. butyricum for microbiota restoration therapy (E3, D6, and C6 in the early phase) were

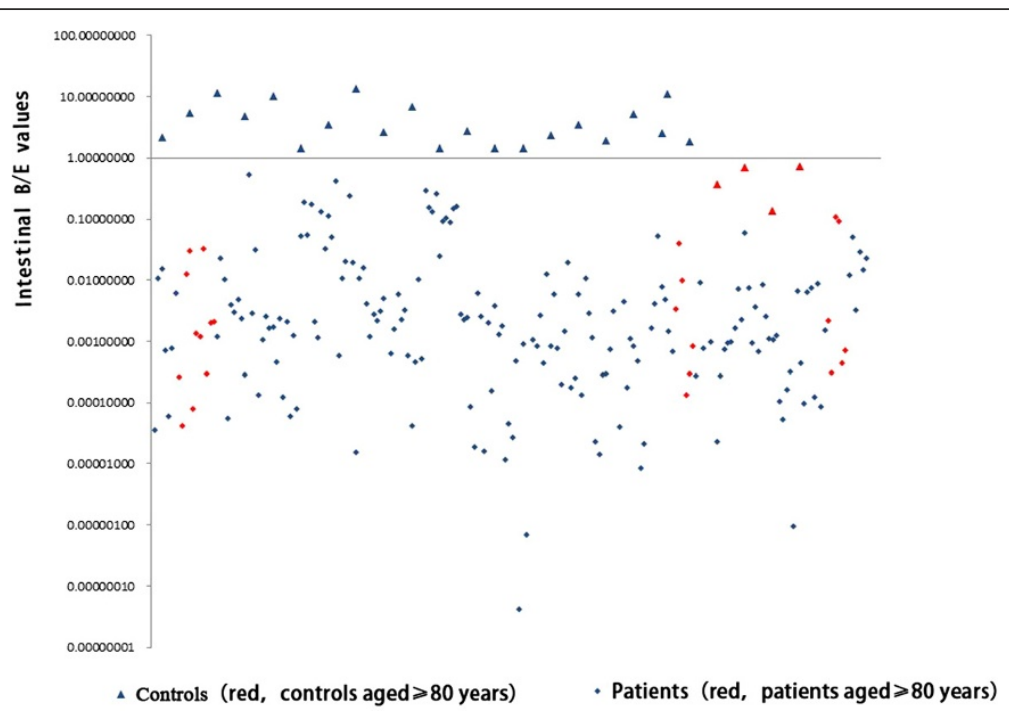

Figure 3 Intestinal B/E ratio in patients with influenza virus H7N9 infection and healthy age- and sex-matched controls. Ratios in most patients were $<1$. Triangles, healthy controls; diamonds, patients. Red symbols denote $\geq 80$ years of age. 


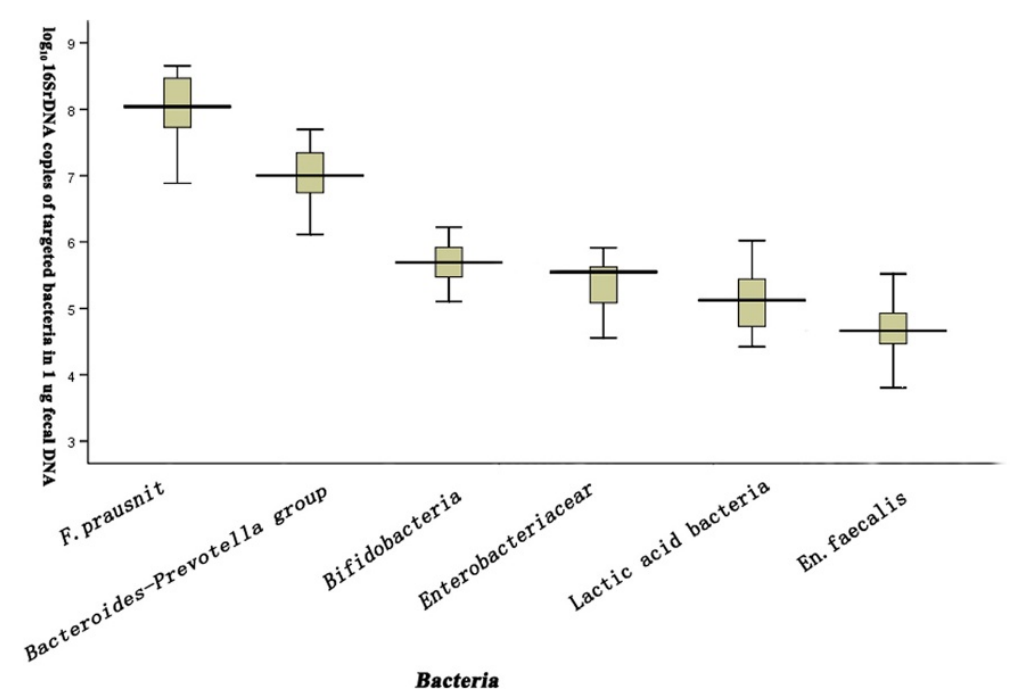

Figure 4 Levels of six predominant intestinal bacterial populations in healthy age- and sex-matched controls.

distributed in the $57-70 \%$ region, which revealed that $C$. butyricum might be involved in the recovery of bacteria with high $\mathrm{GC} \%$ in the V3 region of $16 \mathrm{~S}$ rDNA. Conversely, the profile of $\mathrm{C} 5$ indicated that B. subtilis- and E. faecium-coated enteric capsules might contribute to the restoration of the entire fecal microbiota. In particular, for C6, the most predominant bands were distributed in the $57-70 \%$ region in the early stages with C. butyricum treatment, but were distributed evenly throughout the entire gels when CBM588 was replaced by B. subtilis and E. faecium capsules in the middle stage. Furthermore, the profiles of most patients during the follow-up periods showed relative stability under the continual suppression by the same antibiotics. In contrast, the fecal DGGE profiles of E3 and E8 changed dramatically when new antibacterial agents were introduced during the microbiota restoration therapy.

PCA analysis of DGGE profiles of all patients showed that the predominant intestinal microbiome of groups $\mathrm{D}$ and E differed from groups A, B, and C (Figure 6).

\section{Discussion}

The gastrointestinal tract harbors more than 400 bacterial species, each having a specific niche and playing important roles in human health and disease [20]. Although it is difficult to define the normal intestinal microbiota, there is much evidence suggesting that the balance of beneficial and harmful bacteria (including opportunistic pathogens) is disturbed in many disease states [21-23]. Analysis of the fecal microbiota of healthy controls using DGGE in this study revealed that healthy gut microbiota was characterized by a relatively even distribution and high species richness and diversity. Most patients with influenza virus H7N9 infection had already received antibiotics in the early stages of the disease, and some of them had received repeated antibiotic therapy for secondary infections or prophylaxis against healthcare-associated infections during the microbiota restoration treatment period. In general, the more critically ill the patients were, the more antibiotics were used. Much evidence suggests that antibiotics may cause a profound imbalance within the intestinal microbiota [5] and lead to complications. Therefore, administering beneficial intestinal bacteria during selective decontamination of the intestinal microbiome was recommended by the therapy group in the present study. We monitored the populations of the six predominant intestinal bacterial groups, including three potential pathogens (Enterobacteriaceae, Bacteroides-Prevotella group, and E. faecalis), and three beneficial bacteria (Bifidobacterium species, $F$. prausnitzii, and lactic acid bacteria). The $\mathrm{B} / \mathrm{E}$ values in this study revealed most young and elderly adult patients with H7N9 infection had imbalances between Bifidobacterium and Enterobacteriaceae. The disequilibrium could be caused by many factors, such as the aging process, influenza virus infection, antibiotics, and anorexia. Our results showed that Bifidobacterium was most sensitive to antibioticsuppressionand recovered poorly, even without antibiotic intervention. Additionally, the increasing antimicrobial drug resistance among Enterobacteriaceae [24] may largely account for the disequilibrium. Bifidobacterium inhibits the growth of various pathogens by several mechanisms, including production of antimicrobial agents and short-chain fatty acids, and competitive exclusion [25,26]. Thus, these species play an important role in preventing colonization of the intestine by pathogens. Furthermore, the Bifidobacterium population was often higher than or equal to 

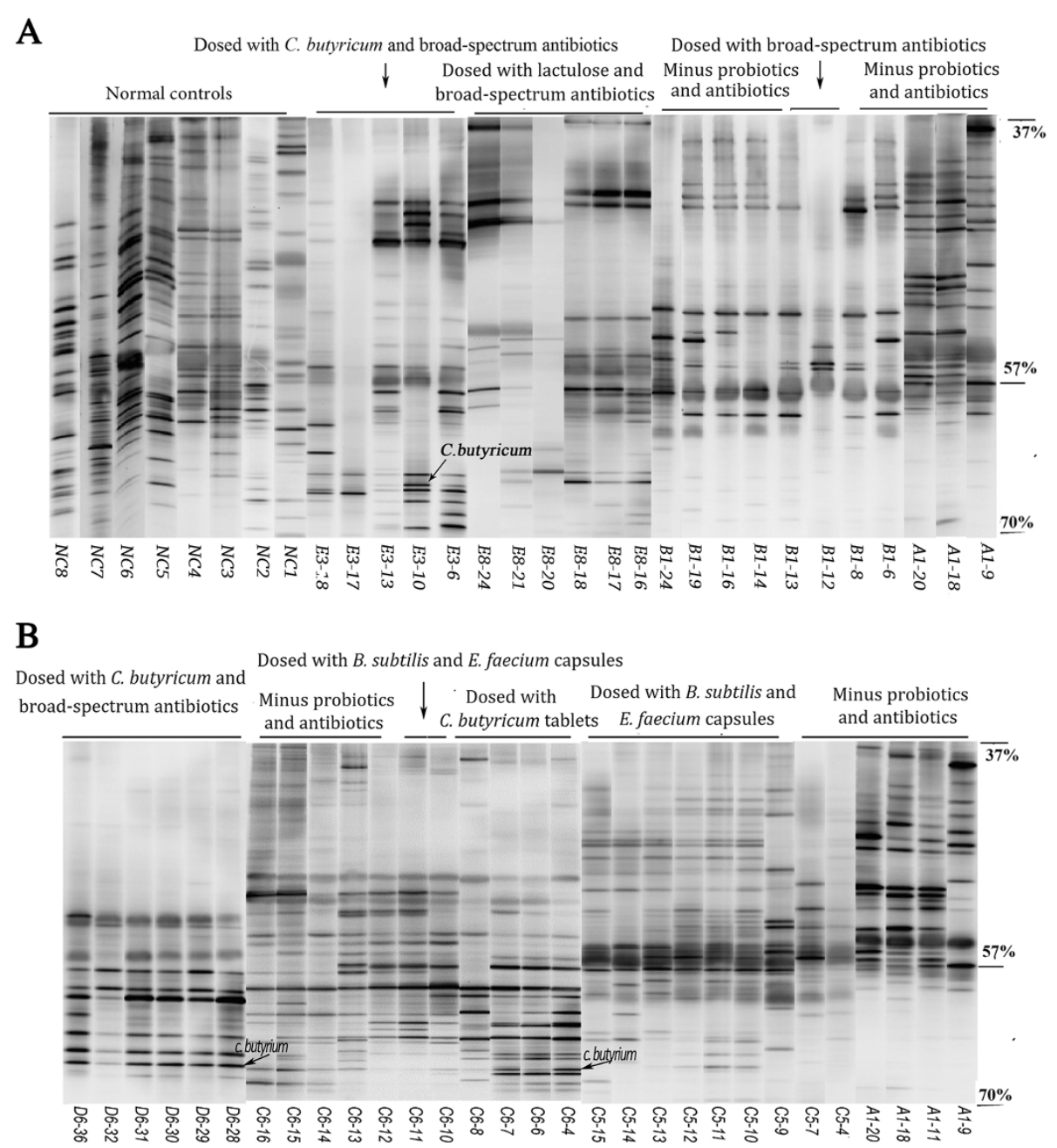

Figure 5 DGGE profiles of representative predominant intestinal bacteria from each group (A1, B1, E3, E8, C5, C6, and D6) and seven healthy controls (NC1-NC7, age- and sex-matched with A1, B1, E3, E8, C5, C6, and D6, respectively). The approximate denaturant concentrations in the gels were $37 \%, 57 \%$, and $70 \%$.

that of the Enterobacteriaceae in most of the healthy controls in this and our previous study [23]. Our previous research showed that the $\mathrm{B} / \mathrm{E}$ ratio is useful for monitoring microecological disruption in the intestine during the progression of liver disease [23].

Secondary infections in most critically ill patients were caused by exogenous rather than endogenous bacteria, most of which were respiratory infections caused by Candida species and multidrug-resistant $A$. baumannii and K. pneumoniae. Previous in vitro and in vivo studies have revealed that bacterial adherence to the surface of cells in the respiratory tract is enhanced by influenza virus infection, which contributes to the increased incidence of secondary bacterial infection in influenza patients [27]. As a result, microecologically targeted therapy was initiated to increase beneficial bacteria and reduce the population of potential pathogens to enhance resistance to intestinal colonization. Probiotics have been proposed to have the potential to modulate host innate immune responses outside the gastrointestinal tract, including the respiratory mucosa [28,29]. Furthermore, probiotic prophylaxis of ventilator-associated pneumonia wasreported to be successful [30]. The relative stability in the profiles of most patients under the continual suppression by the same antibiotics suggested that microbiota restoration therapy with probiotics might help to maintain the relative stability of the microbial community during the period of antibiotic treatment. We also observed that the effect of a combination of antibiotics and pre- or pro-biotic intervention on the predominant intestinal microbiome differed from that of antibiotic or probiotic intervention alone. Individual risk of infection is determined by the relationship between an individual's epidemiological exposure and net state of immunosuppression. A weakened immune system in elderly patients might account for the severity of 


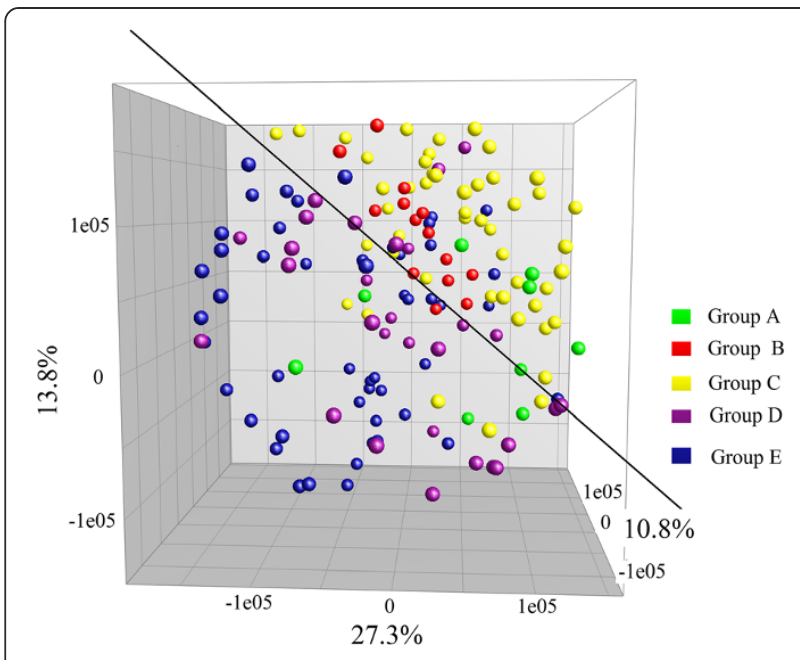

Figure 6 Principal components analysisfor all DGGE profiles of patients. Colored dots show the fecal microbiome profiles of five groups during follow-up. The plot is reoriented to maximize the variation among lanes along the first three principal components (contributions of 27.3, 13.8, and 10.8\%, respectively) obtained from BioNumerics software.

influenza infection and serious complications [31]. However, prophylactic use of probiotics was ineffective in elderly and critically ill patients in this study, and the elderly were stillat particularly high risk for developing secondary bacterial infection as a result of the primary influenza and the complex antibiotic therapies. The artificial liver technology largely contributed to the failure of pro- or pre-biotics to prevent respiratory infections in these patients. Artificial liver technology was used to remove inflammatory cytokines induced by endotoxins because of the use of broad-spectrum antibiotics, which was later confirmed to be a risk factor for critically ill H7N9 patients. In any case, our data showed that CBM588 failed to prevent respiratory infections in elderly and critically ill patients, and its effect on increasing levels of Bifidobacterium in the gut was unsatisfactory. There is a clear need for large volunteer studies on effective prophylaxis strategies for secondary respiratory infections in these elderly patients.

Results from this study demonstrated a transient stability of the intestinal microbiota under continual suppression with the same antibiotics in those patients treated with probiotics. Perhaps the most interesting results in our study were the different profiles of the intestinal microbiome that were restored by C. butyricum, B. subtilis, and E. faecium treatment. Bacillus subtilis- and E. faecium-coated capsules seemed to promote an even distribution in the fecal microbiota, and contribute to restoration of the entire fecal microbiota following antibiotic intervention. Microbiota restoration strategies with multiple probiotics may be more effective than single probiotics in restoring the entire intestinal microbiome. Future studies using properly designed clinical trials are required to support such claims and to uncover the mechanisms. Lactic acid bacteria also have protective effects against bacterial and viral infections in the gastrointestinal and respiratory systems [32], and likely contribute to the accelerated recovery of the innate immune response [28]. The qPCR results in this study showed that lactic acid bacteria recovered more quickly than the Bifidobacteria population, even under antibiotic suppression, which might be beneficial to H7N9 patients.

Another aim of probiotic treatment is to ameliorate inflammation in these patients. All patients with influenza virus H7N9 infection showed a sharp increase in inflammatory markers in the blood, caused by the primary and secondary infections. There are many reports of antiinflammatory activity of probiotics, including CBM588 $[10,33]$. With regard to the assessment of microecological treatment strategies, treatment of H7N9 infection has previously focused on pathogenicity because of the translocation and virulence of probiotics [34], and conflict between antibiotics that kill bacteria and microecological regulatory agents that promote bacterial growth, so that the negative effects did not outweigh the benefits. CRP is normally present in trace levels in the serum, but increases rapidly in response to various infectious or inflammatory conditions [35]. In this study, we found no marked increase in CRP, and no cases of bacteremia and pneumonia, caused by probiotics in the patients.

Prophylaxis against secondary infection with antibiotics and probiotics is as important as antiviral treatment. It is necessary to integrate human and microbial genomic data sets to analyze the risk of human disease and achieve clinically effective strategies to maintain and promote human health [36]. Prevention and treatment of specific infections in these patients should be dictated by knowledge of the infecting microorganism and its sensitivity to antimicrobial agents, as well as the disruption of gut microflora balance during antibiotic treatment. Future studies on the composition of the dominant intestinal microbial populations in patients are important for successful infection prevention. Unfortunately, the number of patients in the present study was small, and all had received antibiotics prior to being transferred to our hospital. Well-designed animal trialsto investigate the use of CBM588 as an adjunct to enhance resistance to intestinal colonization are also needed.

\section{Conclusions}

Our results demonstrated a potential clinical strategy for microbiota-targeted therapy in patients with influenza virus H7N9 infection. The mortality of critically ill patients in our hospital was lower than in other parts of China, which may be partly because of microbiota regulation therapy to compensate for impaired intestinal colonization resistance and to reduce bacteremia and 
sepsis. This study has noteworthy applications in the development of personalized therapeutic strategies by realtime monitoring of the predominant intestinal bacteria using QPCR and DGGE, which is fast, cheap, and comparatively less laborious than high-throughput sequencing technologies. Additionally, the present study is the first to use intestinal $\mathrm{B} / \mathrm{E}$ ratio for rapid nonintrusive assessment of intestinal colonization resistance and microbiota-regulated therapy in patients with influenza virus $\mathrm{H} 7 \mathrm{~N} 9$ infection.

\section{Abbreviations}

DGGE: Denaturing gradient gel electrophoresis; CRP: C-reactive protein; qPCR: Quantitative polymerase chain reaction; CBM588: Clostridium butyricum 588 strain MIYA-BM tablets.

\section{Competing interests}

The authors declare that they have no competing interests.

\section{Authors' contributions}

$\mathrm{HL}$ and $\mathrm{CZ}$ helped design the study, collected and analyzed data, and drafted the manuscript. GQ and XH performed the experiments and acquired data. ZH prepared the figures. CC, WL, HG and YY had roles in recruitment, field investigation, and were involved in data analysis, interpretation and write-up. LL designed the study and critically revised the manuscript. All authors read and approved the final manuscript.

\section{Acknowledgments}

We acknowledge the physicians at the Department of Infectious Diseases and Intensive Care Unit in the First Affiliated Hospital, College of Medicine, Zhejiang University, for their collaboration and collection of stool specimens. This study was supported by the National Basic Research Program (973) of China (2013CB531401, 2013CB531402), Important National Science and Technology Project-Research of Clinical Treatment for Emerging Severe Acute Respiratory Infectious Disease (2004ZX10004006), the Zhejiang Chinese Medicine Administrative Bureau Project (2009ZA012), and the Zhejiang Provincial Medical Scientific Research Foundation of China (2012RCB020).

\section{Author details}

'State Key Laboratory Diagnosis and Treatment for Infectious Disease, Collaborative Innovation Center for Diagnosis and Treatment of Infectious Diseases, the First Affiliated College of Medicine, Zhejiang University, 79 Qingchun Road, Hangzhou 310003, China. ${ }^{2}$ Collaborative Innovation Center for Diagnosis and Treatment of Infectious Diseases, Hangzhou, China. ${ }^{3}$ Department of Geriatrics, the First Affiliated Hospital, College of Medicine, Zhejiang University, 79 Qingchun Road, Hangzhou 310003, P.R. China.

Received: 13 June 2013 Accepted: 26 June 2014

Published: 2 July 2014

\section{References}

1. Xi XM, Gu Q, Zhou XM, Qu HP, Yan Z, Li FM, Zhao W, Gao ZC, Wang GF, Ruan LX, Wang WH, Ye J, Cao HF, Li XW, Zhang WH, Fang XC, He J, Liang WF, Xie J, Zeng M, Wu XZ, Li J, Xia Q, Jin ZC, Chen Q, Tang C, Zhang ZY, Hou BM, Feng ZX, Sheng JF, et al: Clinical findings in 111 cases of influenza a (H7N9) virus infection. N Engl J Med 2013, 68(24):2277-2285.

2. Clemente JC, Ursell LK, Parfrey LW, Knight R: The impact of the gut microbiota on human health: an integrative view. Cell 2012 148(6):1258-1270.

3. Hemarajata P, Versalovic J: Effects of probiotics on gut microbiota: mechanisms of intestinal immunomodulation and neuromodulation. Therap Adv Gastroenterol 2013, 6(1):39-51.

4. Andremont A: Consequences of antibiotic therapy to the intestinal ecosystem. Ann Fr Anesth Reanim 2000, 19(5):395-402.

5. Sekirov I, Tam NM, Jogova M, Robertson ML, Li Y, Lupp C, Finlay BB: Antibiotic-induced perturbations of the intestinal microbiota alter host susceptibility to enteric infection. Infect Immun 2008, 76(10):4726-4736.
6. Fujimura KE, Slusher NA, Cabana MD, Lynch SV: Role of the gut microbiota in defining human health. Expert Rev Anti Infect Ther 2010, 8(4):435-454.

7. Lawley TD, Walker AW: Intestinal colonization resistance. Immunology 2013, 138(1):1-11.

8. Tuohy KM, Probert HM, Smejkal CW, Gibson GR: Using probiotics and prebiotics to improve gut health. Drug Discov Today 2003, 8(15):692-700.

9. Isolauri E, Sutas Y, Kankaanpaa P, Arvilommi H, Salminen S: Probiotics: effects on immunity. Am J Clin Nutr 2001, 73(Suppl 2):444S-450S.

10. Imase K, Takahashi M, Tanaka A, Tokunaga K, Sugano H, Tanaka M, Ishida H, Kamiya S, Takahashi S: Efficacy of clostridium butyricum preparation concomitantly with helicobacter pylori eradication therapy in relation to changes in the intestinal microbiota. Microbiol Immunol 2008, 52(3):156-161.

11. Okamoto T, Sasaki M, Tsujikawa T, Fujiyama Y, Bamba T, Kusunoki M: Preventive efficacy of butyrate enemas and oral administration of clostridium butyricum M588 in dextran sodium sulfate-induced colitis in rats. J Gastroenterol 2000, 35(5):341-346.

12. Chinese Center for Disease Control and Prevention: Diagnostic and treatment protocol for human infections with avian influenza a (H7N9). http://www.chinacdc.cn/en/research_5311/Guidelines/201304/ W020130425363145259626.pdf.

13. Bartosch S, Fite A, Macfarlane GT, McMurdo ME: Characterization of bacterial communities in feces from healthy elderly volunteers and hospitalized elderly patients by using real-time PCR and effects of antibiotic treatment on the fecal microbiota. Appl Environ Microbiol 2004, 70(6):3575-3581.

14. Bartosch B, Verney G, Dreux M, Donot P, Morice Y, Penin F, Pawlotsky JM, Lavillette $D$, Cosset $F L$ : An interplay between hypervariable region 1 of the hepatitis $\mathrm{C}$ virus $\mathrm{E} 2$ glycoprotein, the scavenger receptor $\mathrm{Bl}$, and high-density lipoprotein promotes both enhancement of infection and protection against neutralizing antibodies. J Virol 2005, 79(13):8217-8229.

15. Haarman M, Knol J: Quantitative real-time PCR analysis of fecal lactobacillus species in infants receiving a prebiotic infant formula. Appl Environ Microbiol 2006, 72(4):2359-2365.

16. Muyzer G, de Waal EC, Uitterlinden AG: Profiling of complex microbial populations by denaturing gradient gel electrophoresis analysis of polymerase chain reaction-amplified genes coding for 16S rRNA. Appl Environ Microbiol 1993, 59(3):695-700.

17. Thompson JR, Marcelino LA, Polz MF: Heteroduplexes in mixed-template amplifications: formation, consequence and elimination by 'reconditioning PCR'. Nucleic Acids Res 2002, 30(9):2083-2088.

18. Joossens M, Huys G, Cnockaert M, De Preter V, Verbeke K, Rutgeerts P, Vandamme $P$, Vermeire $S$ : Dysbiosis of the faecal microbiota in patients with Crohn's disease and their unaffected relatives. Gut 2011, 60(5):631-637.

19. Lu H, He J, Wu Z, Xu W, Zhang H, Ye P, Yang J, Zhen S, Li L: Assessment of microbiome variation during the perioperative period in liver transplant patients: a retrospective analysis. Microb Ecol 2013, 65(3):781-791.

20. Hart AL, Stagg AJ, Frame M, Graffner H, Glise H, Falk P, Kamm MA: The role of the gut flora in health and disease, and its modification as therapy. Aliment Pharmacol Ther 2002, 16(8):1383-1393.

21. Chen Y, Yang F, Lu H, Wang B, Chen Y, Lei D, Wang Y, Zhu B, Li L: Characterization of fecal microbial communities in patients with liver cirrhosis. Hepatology 2011, 54(2):562-572.

22. Festi D, Schiumerini R, Birtolo C, Marzi L, Montrone L, Scaioli E, Di Biase AR, Colecchia A: Gut microbiota and its pathophysiology in disease paradigms. Dig Dis 2011, 29(6):518-524.

23. Lu H, Wu Z, Xu W, Yang J, Chen Y, Li L: Intestinal microbiota was assessed in cirrhotic patients with hepatitis B virus infection. Intestinal microbiota of HBV cirrhotic patients. Microb Ecol 2011, 61(3):693-703.

24. Falagas ME, Maraki S, Karageorgopoulos DE, Kastoris AC, Mavromanolakis E, Samonis G: Antimicrobial susceptibility of multidrug-resistant (MDR) and extensively drug-resistant (XDR) Enterobacteriaceae isolates to fosfomycin. Int J Antimicrob Agents 2010, 35(3):240-243.

25. Gibson GR, Wang X: Regulatory effects of bifidobacteria on the growth of other colonic bacteria. J Appl Bacteriol 1994, 77(4):412-420.

26. Lievin V, Peiffer I, Hudault S, Rochat F, Brassart D, Neeser JR, Servin AL: Bifidobacterium strains from resident infant human gastrointestinal microflora exert antimicrobial activity. Gut 2000, 47(5):646-652.

27. Hedlund M, Aschenbrenner LM, Jensen K, Larson JL, Fang F: Sialidasebased anti-influenza virus therapy protects against secondary pneumococcal infection. J Infect Dis 2010, 201(7):1007-1015. 
28. Forsythe P: Probiotics and lung diseases. Chest 2011, 139(4):901-908.

29. Gourbeyre P, Denery S, Bodinier M: Probiotics, prebiotics, and synbiotics: impact on the gut immune system and allergic reactions. J Leukoc Biol 2011, 89(5):685-695.

30. Morrow LE, Kollef MH, Casale TB: Probiotic prophylaxis of ventilatorassociated pneumonia: a blinded, randomized, controlled trial. Am J Respir Crit Care Med 2010, 182(8):1058-1064.

31. Centers for Disease Control and Prevention: People at high risk of developing flu-related complications. http://www.cdc.gov/flu/about/ disease/high_risk.htm.

32. Goldin BR, Gorbach SL: Clinical indications for probiotics: an overview. Clin Infect Dis 2008, 46(Suppl 2):S96-S100.

33. Mengheri E: Health, probiotics, and inflammation. J Clin Gastroenterol 2008, 42(Suppl 3 Pt 2):S177-S178.

34. Boyle RJ, Robins-Browne RM, Tang ML: Probiotic use in clinical practice: what are the risks? Am J Clin Nutr 2006, 83(6):1256-1264.

35. Ballou SP, Kushner I: C-reactive protein and the acute phase response. Adv Intern Med 1992, 37:313-336.

36. Grice EA, Segre JA: The human microbiome: our second genome. Annu Rev Genomics Hum Genet 2012, 13:151-170.

doi:10.1186/1471-2334-14-359

Cite this article as: Lu et al:: An analysis of microbiota-targeted therapies in patients with avian influenza virus subtype H7N9 infection. BMC

Infectious Diseases 2014 14:359.

\section{Submit your next manuscript to BioMed Central and take full advantage of:}

- Convenient online submission

- Thorough peer review

- No space constraints or color figure charges

- Immediate publication on acceptance

- Inclusion in PubMed, CAS, Scopus and Google Scholar

- Research which is freely available for redistribution 